\title{
Which criteria should be used to select patients for the Fontan operation?
}

\author{
Melvin C Almodovar ${ }^{1}$ and Leonardo Mulinari ${ }^{1}$ \\ ${ }^{1}$ University of Miami Miller School of Medicine
}

December 17, 2020

\begin{abstract}
The Fontan operation has improved the survival of children born with single ventricle physiology. Selecting candidates for the Fontan operation may be difficult on borderline cases. No clear criterion has been established on the risk for staged Fontan palliation. Another aspect that remains controversial is the indications for fenestration. Intraoperative pulmonary flow study may identify high-risk patients for the procedure. In this report, the authors describe their results with Fontan procedures in children with pulmonary pressure $>15 \mathrm{mmHg}$.
\end{abstract}

Title

Which criteria should be used to select patients for the Fontan operation?

Authors

Melvin Almodovar

University of Miami Miller School of Medicine, Pediatrics

1611 SW 12th Ave

Miami, FL, USA 33136-1015

melalmodovar@med.miami.edu

Leonardo Mulinari

(Corresponding Author)

University of Miami Miller School of Medicine, Surgery

1611 NW 12th Ave

East Tower Suite 3016A

Miami, FL, USA 33136-1015

l.mulinari@med.miami.edu

Data sharing is not applicable to this article as no new data were created or analyzed in this study.

Funding: none

Conflict of interest: none

The Fontan operation has improved the survival of children born with congenital heart disease with single ventricle physiology. Long-term survival of patients undergoing Fontan operation is limited due to ventricular 
dysfunction, protein-losing enteropathy, plastic bronchitis, all contributing to the failure of the procedure. Selecting candidates for the Fontan operation may sometimes be difficult in borderline cases. No clear criterion has been established on the risk of staged Fontan palliation. Another aspect that remains controversial is the indication for fenestration. Intraoperative flow pulmonary flow study may identify the high-risk cases.11Baek JS, Park CS, Yun TJ, Bae EJ. Identification of high-risk Fontan candidates by intraoperative pulmonary flow study. Int J Cardiol. 2020 Sep 25:S0167-5273(20)33840-7. doi: 10.1016/j.ijcard.2020.09.059. Epub ahead of print. PMID: 32987053.

Özyüksel et al describe a retrospective analysis of their results with Fontan patients who have preoperative pulmonary artery pressure $>15 \mathrm{mmHg}$. 22Özyüksel A, Şimşek B, Özden Ö2, et al. Fontan Procedure in Patients with Preoperative Mean Pulmonary Artery Pressure Over 15 mmHg. Journal of Cardiac Surgery in press

The major indications for the Fontan procedures are patients with low pulmonary vascular resistance (PVR), determined by cardiac catheterization $(<3-3.5 \mathrm{WU})$, and with preserved ventricular function (both systolic and diastolic). Meeting these requirements involves addressing at time of prep operative catheterization, or in the operating room, the following: peripheral pulmonary stenosis, systemic outflow obstruction, assuring unobstructed atrial communication in hypoplastic left heart cases, reducing aortopulmonary collaterals (APC), systemic vein to pulmonary vein connections, atrioventricular valve (AVV) regurgitation, and assuring atrioventricular synchrony.

Analyzing pulmonary artery pressure per se may not describe PVR accurately, as some patients have a significant burden of APC's, antegrade flow (pulsatile Glenn), elevated end-diastolic pressure (EDP) due to AVV regurgitation, or systemic outflow obstruction, which may be correctable at time of surgery. Even one lung patient may be suitable for Fontan if PVR is low. 33Fujii Y, Sano S, Asou T, Imoto Y, Oshima Y, Kawasaki S, Kishimoto H, Sakamoto K, Maeda M, Yamagishi M, Matsuo K. Outcomes of one-lung Fontan operation: a retrospective multicenter study in Japan. Ann Thorac Surg. 2012 Oct;94(4):1275-80; discussion 1280. doi: 10.1016/j.athoracsur.2012.04.080. Epub 2012 Jul 6. PMID: 22771056.

We believe all patients with any risk factor for difficulty after the Fontan procedure should have fenestration. Even low-risk patients may develop low cardiac output or prolonged hospital stay due to pleural effusions that could have benefited from a fenestration.44Bradley SM. Use of a fenestration should be routine during the Fontan procedure: PRO. Semin Thorac Cardiovasc Surg Pediatr Card Surg Annu. 2010;13(1):55-9. doi: 10.1053/j.pcsu.2010.01.004. PMID: 20307862. Patients that fail typically have high transpulmonary gradient with low cardiac output in the operating room, or within the first 24-48 hours. During this time, maneuvers to reduce PVR while maintaining ventricular preload can be tried. If no improvement after open fenestration, pulmonary vasodilators, careful ventilation (most such patients will still be intubated) AV synchrony, adequate ventricular function, fluid resuscitation, or the Fontan may have to be taken down. Perhaps the most important sign of failure is low cardiac output plus ascites.

In this series reported by Özyüksel et al, the main inclusion criterion for patients was PA pressure, not resistance, or another measure to describe the pulmonary vascular cross-sectional area (PVR, Nakata Index, etc.). Another aspect is that $30 \%$ of the patients had additional flow source to the Glenn (i.e., antegrade flow with PA band, BT shunt). This could contribute to higher PA pressure but may also contribute to PA growth. The patients were older at the time of Fontan (mean 8 years with large standard deviation) and had a long interstage period from Glenn. This meant they had a longer period of cyanosis. All had lower or similar PA or Fontan pressures compared to preoperative. What is remarkable is that hospital stay was short, and there were no mortalities or takedowns, and prolonged pleural drainage was not a problem despite the use of fenestration in only $31 \%$.

The reason for a favorable outcome is that PVR and pulmonary cross-sectional area was actually better than one might anticipate when looking at PA pressure alone. Alternatively, maybe these patients did have PVR slightly higher than our current upper limit (3-4 WU), and they did well because of other factors such as intraoperative management, post-operative management, avoiding complications like phrenic nerve injury, 
etc. This suggests that one can be more permissive in selecting Fontan candidates, but the data would not be compelling. In other words, the standard approach to selecting Fontan candidates has been so successful with current criteria that we would need a more robust prospective study to change that paradigm.

The authors use routinely Sildenafil and Bosentan postoperatively which may have a positive effect in lowering PVR after the immediately post-period until the systemic venous and pulmonary circulations adjust to the Fontan completion.

Finally, the questions remain: What is the highest preoperative PA pressure that is considered acceptable for a patient to proceed with Fontan completion? We respond that we should not make the decision based on PA pressure, but instead go to lengths necessary to assess PVR, pulmonary cross-sectional area (distally) along with objective measures of ventricular function (systolic and diastolic) as early in life as possible to correct cyanosis. It is also important to consider other factors that affect the respiratory system in both the short and long-term. 